Review article

\title{
The efficacy of biofeedback approaches for obsessive-compulsive and related disorders: A systematic review and meta-analysis
}

\author{
Sónia Ferreira ${ }^{\mathrm{a}, \mathrm{b}}$, José Miguel Pêgo ${ }^{\mathrm{a}, \mathrm{b}}$, Pedro Morgado ${ }^{\mathrm{a}, \mathrm{b}, *}$ \\ ${ }^{\mathrm{a}}$ Life and Health Sciences Research Institute (ICVS), School of Medicine, University of Minho, Braga, Portugal \\ b ICVS/3B's - PT Government Associate Laboratory, Braga/Guimarães, Portugal
}

\section{A R T I C L E I N F O}

\section{Keywords:}

Neurofeedback

Obsessive-compulsive disorder

Self-regulation

Treatment outcome

Functional magnetic resonance imaging

Electroencephalography

Human

\begin{abstract}
A B S T R A C T
Biofeedback is applied to target excessive and/or deficient physiological signals to help patients identifying and self-managing their symptoms. Biofeedback has been employed in psychiatric disorders, including obsessivecompulsive disorder (OCD), mainly by using neural signals - neurofeedback. Recently, OCD has been integrated into the obsessive-compulsive and related disorders (OCD\&RD) category (body dysmorphic, hoarding, trichotillomania/hair-pulling, and excoriation/skin-picking disorders). The efficacy of biofeedback for OCD\&RD is still unknown. Our work provides a complete overview of publications assessing the therapeutic efficacy of biofeedback in OCD\&RD with a systematic review and meta-analysis. We found ten studies involving 102 OCD participants (three randomized controlled trials) mostly applying neurofeedback (one publication used thermal biofeedback). Five neurofeedback studies were selected for meta-analysis (89 patients; two randomized controlled trials). The overall effect size within the treatment group varied between medium to large, but high heterogeneity and inconsistency values were found. The methodological quality was low indicating a high risk of bias. In conclusion, a beneficial effect of neurofeedback for OCD patients was found but also critical limitations on methodology, high heterogeneity among studies, and a putative reporting bias. Future research following high-quality guidelines should be conducted to address the efficacy of biofeedback approaches for OCD\&RD.
\end{abstract}

\section{Introduction}

OCD is the fourth most common psychiatric disorder and the tenth cause of disability worldwide. OCD is characterized by recurrent thoughts (obsessions) associated with high anxiety, followed by repetitive behaviors or mental tasks to relieve the anxiety (compulsions) (Rapp et al., 2016; Sachs and Erfurth, 2018). Anxiety symptoms comprise autonomic nervous system dysregulation namely increased heart rate, decreased heart rate variability, elevated skin electrodermal activity, and augmented breathing rate (Schoenberg and David, 2014; Simon et al., 2013). OCD is also characterized by a hyperactive orbitofronto-striatal circuit including the orbitofrontal and cingulate cortices and subcortical structures (basal ganglia, hippocampus, and amygdala) (Bruin et al., 2018; Moreira et al., 2017). Recently, OCD has been integrated into the OCD\&RD category in the fifth edition of the Diagnostic and Statistical Manual of Mental Disorders (DSM-5). This category includes OCD, body dysmorphic disorder, hoarding disorder, trichotillomania/hair-pulling disorder, excoriation/skin-picking disorder, substance/medication-induced OCD\&RD, and OCD\&RD induced by other medical condition. These disorders share compulsive behaviors, impaired behavioral inhibition, demographic characteristics (onset age, family patterns, and comorbidity), neural pathways (e.g. increased basal ganglia activity) and neurotransmitter dysfunction (serotonergic and dopaminergic systems imbalance), and treatment response profiles (Abramowitz, 2018; American Psychiatric Association, 2013).

First-line treatments include cognitive-behavioral therapy (CBT) and antidepressants. However, up to $60 \%$ of patients do not present symptomatic remission after treatment (Seibell and Hollander, 2014). The limited success of conventional treatments makes identifying alternative therapies a priority. Self-regulation is the ability to manage emotions, thoughts, or behaviors in face of specific stimuli

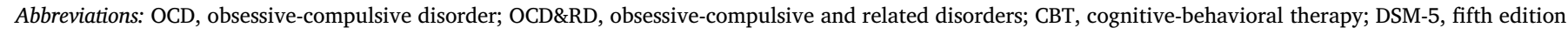

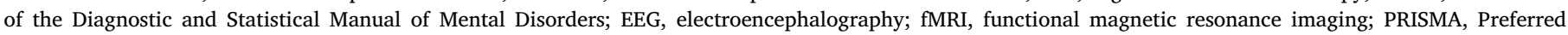

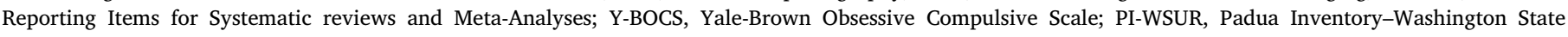
University Revision

* Corresponding author at: Escola de Medicina, Universidade do Minho, Campus de Gualtar, 4710-057 Braga, Portugal.

E-mail addresses: id6533@alunos.uminho.pt (S. Ferreira),jmpego@med.uminho.pt (J.M. Pêgo), pedromorgado@med.uminho.pt (P. Morgado).
} 
(Pearcy et al., 2016). Biofeedback is a technique aiming to inhibit excessive and/or reinforce impaired physiological signals to help patients in identifying and managing their symptoms. It is also applied to improve the performance or cognitive skills. Usually, biosignal changes are measured and presented to patients in form of auditory or visual feedback so they can learn how to self-regulate their biological responses. These signals might be related to heart rate, skin conductance, temperature levels, respiratory pattern, or muscle activity (Schoenberg and David, 2014).

Biofeedback has been applied to psychiatric disorders such as anxiety, depression, and schizophrenia with a specific type of feedback from neural activity in target brain regions - neurofeedback (Schoenberg and David, 2014; Sitaram et al., 2016). The neurofeedback technique is mostly implemented with functional magnetic resonance imaging (fMRI) or electroencephalography (EEG) (Begemann et al., 2016; Sitaram et al., 2016; Thibault et al., 2018).

Previous authors have explored the efficacy of biofeedback (Schoenberg and David, 2014) and neurofeedback (Begemann et al., 2016; Gonçalves et al., 2017; Micoulaud-Franchi et al., 2015) in psychiatric disorders, including OCD. These works described that EEG neurofeedback reduces OCD rumination and anxiety (Schoenberg and David, 2014) and has a superior effect compared to sham neurofeedback (false feedback signal used as a placebo condition) (MicoulaudFranchi et al., 2015). Moreover, EEG neurofeedback effects were reported to be similar to medication, mainly for reducing compulsions (Begemann et al., 2016). Lastly, OCD patients learned how to regulate brain regions (the anterior insula and orbitofrontal cortex) with fMRI neurofeedback leading to clinical improvement (Gonçalves et al., 2017).

However, the efficacy of biofeedback techniques for the OCD\&RD is still unknown. Prior studies have mainly focused on neurofeedback approaches and OCD. It is important to explore the literature to establish adequate biofeedback protocols to maximize the clinical outcomes for patients. This work aims to review the evidence for the therapeutic efficacy of biofeedback in OCD\&RD. Our work provides qualitative and quantitative information to evaluate if biofeedback is a viable therapeutic approach for OCD\&RD.

\section{Methods}

This review was performed according to the Preferred Reporting Items for Systematic Reviews and Meta-Analyses (PRISMA) norms (Liberati et al., 2009; Moher et al., 2009). The study protocol was not registered at PROSPERO (Centre for Reviews and Dissemination, York, U. K., https://www.crd.york.ac.uk/prospero).

\subsection{Eligibility criteria}

All studies regarding the efficacy of biofeedback techniques in the reduction of symptomatic manifestation in OCD\&RD were considered. Only studies conducted with humans, published in English, and reporting original results were selected (conference abstracts, reviews, and book chapters were excluded).

\subsection{Information sources}

Studies were identified by searching electronic databases and reference lists of articles. This search was last conducted on 27th of August 2018 in Medline, Web of Science, Scopus, and PsycINFO without any date restrictions. The search was conducted by the author SF.

\subsection{Search}

The following setup of search terms was used for Medline and similar terms were used for the other databases: ("obsessive-compulsive disorder" OR "body dysmorphic disorders" OR "hoarding disorder" OR "trichotillomania" OR "hair-pulling disorder" OR "excoriation disorder" OR "skin-picking disorder") AND ("neurofeedback" OR "biofeedback").

\subsection{Study selection}

Eligibility assessment was performed by the author SF. In case of doubt, the results were discussed among all authors. All trials were included, regardless of the existence and type of a comparator group. The primary outcome measure was the effect of biofeedback on symptomatic manifestation based on psychometric scales. Articles including participants with obsessive-compulsive tendencies or symptoms without a diagnosis were excluded. Moreover, studies with several disorders including OCD\&RD that did not present the results for each condition individually were also excluded. Articles that did not use biofeedback as a therapeutic approach were also excluded. Works not assessing symptomatic changes after biofeedback application were also excluded.

\subsection{Data collection process}

We develop a data extraction sheet based on a previous review article (Schoenberg and David, 2014) and refined it to simplify the organization of information. All data extracted by the author SF was confirmed twice to avoid errors. In case of doubt, the results were discussed among all authors. Studies from the same research group or group of authors were carefully analyzed to avoid double counting the same data.

\subsection{Data items}

From each study, we extracted the following data items: (1) participant groups [sample size, average age, and gender ratio]; (2) disorder [type, method of diagnosis, previous/current treatment approaches, and associated comorbidities]; (3) biofeedback intervention [type of biosignal, number of sessions, session duration, and outcome measures].

\subsection{Risk of bias in individual studies}

To ascertain the risk of bias of the eligible studies, the author SF determined the quality of each study concerning study control, randomization, patients' blindness, researchers' blindness, and sponsoring bias. Three levels were used for evaluating each parameter: low, moderate, and high quality (Higgins and Green, 2008; Liberati et al., 2009).

\subsection{Synthesis of results}

For each study, the mean score and standard deviation of psychometric scales at baseline and after biofeedback intervention were extracted or calculated from median values (Hozo et al., 2005) or individual data. Only scales reflecting the severity of symptoms were considered. The effect size of the intervention was determined based on the standardized mean change. Since individual data was not available in most of the studies, we considered a correlation coefficient $(r)$ of 0.6 between pre and post-intervention measures (Morris, 2008; Morris and DeShon, 2002; Nakagawa and Cuthill, 2007) [the supplementary Figure S.1 and Figure S.2 represent the main results for a lower $r=0.1$ and higher $r=0.9$, respectively]. Studies reporting only qualitative results, insufficient data for the effect size calculation, and low sample sizes preventing statistical analysis ( $n<3$ per group) were excluded.

Heterogeneity (Cochrane's $Q$ ) and inconsistency $\left(I^{2}\right)$ values were also calculated (Hardy and Thompson, 1998; Higgins, 2003; Higgins and Thompson, 2002). Ninety-five percent confidence intervals (CI) were considered. All calculations and graphs were executed in $\mathrm{R}$ version 


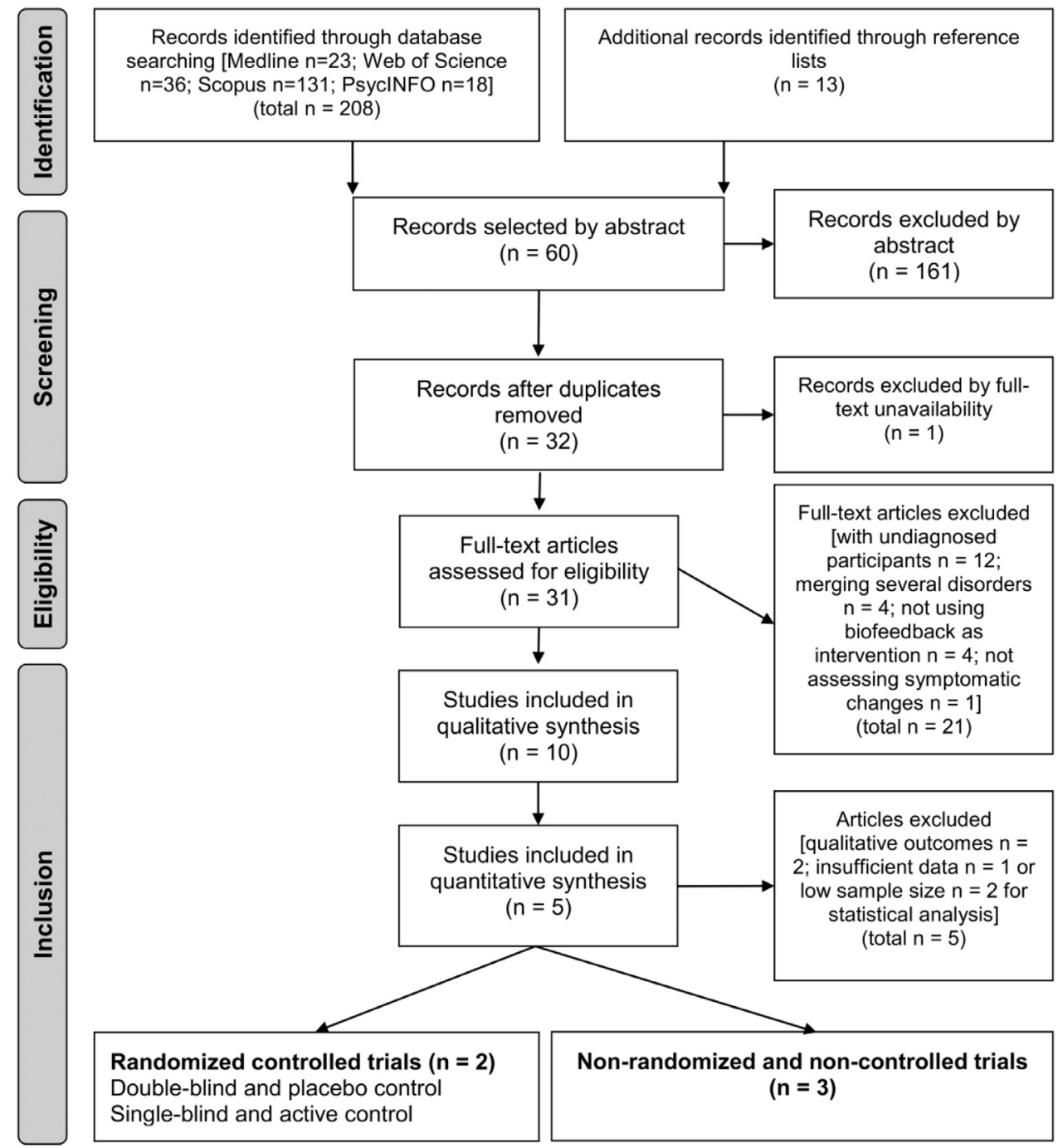

Fig. 1. Flow diagram of the literature search [adapted from (Liberati et al., 2009; Moher et al., 2009)].

3.4.3 (package "metaphor", The R Foundation, https://www.r-project. org/) using a random effects model.

\subsection{Risk of bias across studies}

To assess bias across studies, a funnel plot was constructed. Given the small number of studies included for meta-analysis (Fig. 1), the Egger's test was not performed to evaluated funnel plot asymmetry (Sterne et al., 2011), thus, asymmetry was visually inspected.

\subsection{Additional analyses}

To evaluate if the effect size heterogeneity was explained by the patients' characteristics in each study, an exploratory meta-regression was performed using the mean age and gender ratio (females/males proportion) as predictors (Table 1). Additionally, the mean number of biofeedback sessions was also introduced as a predictor to analyze if the effect size depended on treatment duration (session duration information was not available for all studies, preventing the calculation of treatment duration in time; Table 2) (Thompson and Higgins, 2002).

\section{Results}

\subsection{Study selection}

Fig. 1 shows the flow diagram representative of the process of studies selection. A total of 221 studies were identified through databases searching $(n=208)$ and by checking relevant articles in reference lists ( $n=13)$. No unpublished articles were found. From those, 32 studies were selected to be included in the review after reading the abstract and removing duplicates. From those, 1 study was discarded because the full text was not available even after contacting the authors. The remaining 31 articles were examined in detail. Twenty-one studies did not meet the inclusion criteria: studies including participants without a diagnosis of OCD\&RD $(n=12)$, studies presenting results for a group merging several disorders including OCD\&RD $(n=4)$, articles not using the biofeedback technique as a therapeutic intervention $(n=4)$, and studies not assessing symptomatic changes associated with OCD\&RD $(n=1)$.

\subsection{Study characteristics}

All studies included in the systematic review $(n=10)$ involved OCD patients and were published between 1974 and 2015. Two multicentric studies were conducted in Germany, Italy, USA, and Chile (Buyukturkoglu et al., 2015), and the Czech Republic and France 


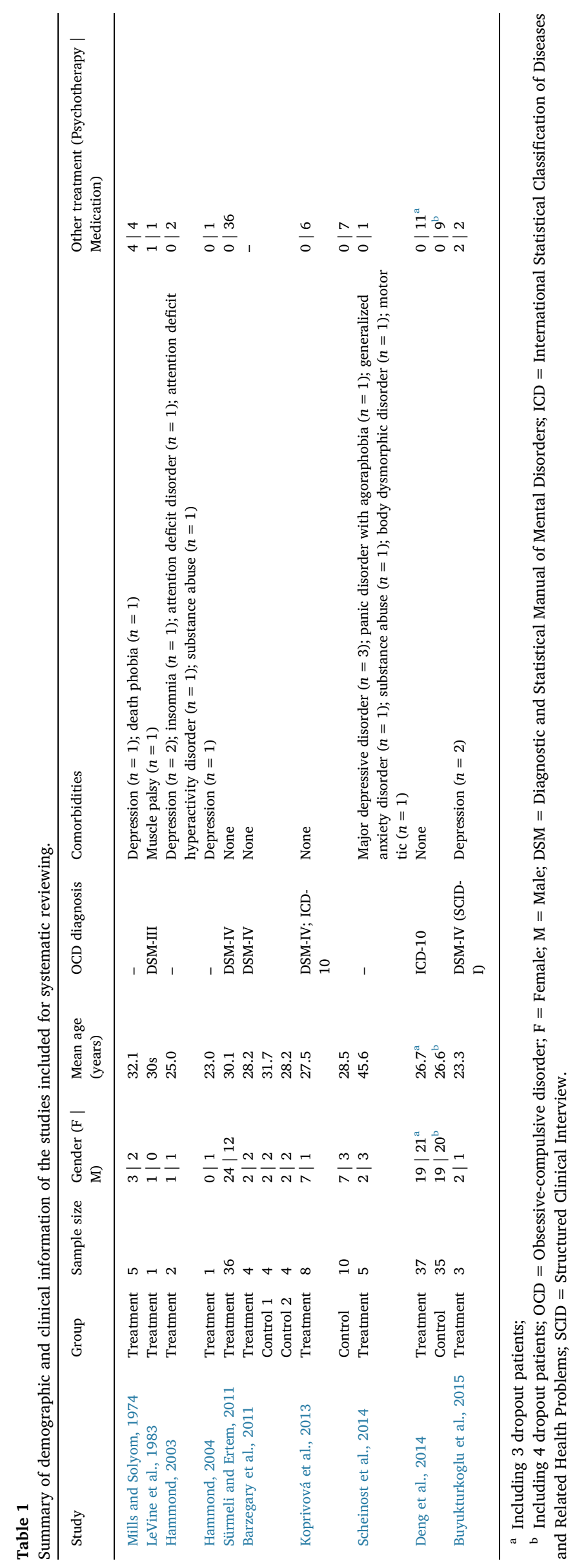


Table 2

Summary of the biofeedback intervention information of the studies included for systematic reviewing and meta-analysis.

\begin{tabular}{|c|c|c|c|c|c|c|c|}
\hline Study & Group & Biofeedback intervention & Biosignal & $\begin{array}{l}\text { Mean number } \\
\text { of sessions }\end{array}$ & $\begin{array}{l}\text { Session } \\
\text { duration ( } \mathrm{min} \text { ) }\end{array}$ & $\begin{array}{l}\text { Outcome } \\
\text { measure }\end{array}$ & $\begin{array}{l}\text { Effect size within } \\
\text { treatment group [CI } \\
95 \%]\end{array}$ \\
\hline Mills and Solyom, 1974 & Treatment & EEG & $\begin{array}{l}\text { EEG alpha at } \mathrm{O} 1 \text { and } \mathrm{O} 2 \\
(8-13 \mathrm{~Hz} ;>20 \mu \mathrm{v})\end{array}$ & 15.2 & 60 & - & Qualitative outcomes \\
\hline LeVine et al., 1983 & Treatment & Thermal & Hand fingers temperature & - & 30 & - & Qualitative outcomes \\
\hline Hammond, 2003 & Treatment & $\mathrm{qEEG}$ & Individualized EEG & 71.5 & $15-35$ & Y-BOCS & Low sample size \\
\hline Hammond, 2004 & Treatment & qEEG & Individualized EEG & 43.0 & 30 & Y-BOCS & Low sample size \\
\hline Sürmeli and Ertem, 2011 ${ }^{\mathrm{a}}$ & Treatment & qEEG & Individualized EEG & 50.2 & 60 & Y-BOCS & $2.18[1.60 ; 2.77]$ \\
\hline \multirow[t]{3}{*}{ Barzegary et al., 2011} & Treatment & qEEG & Individualized EEG & 30.0 & 45 & \multirow[t]{3}{*}{ PI-WSUR } & \multirow[t]{3}{*}{ Insufficient data } \\
\hline & Control 1 & Drug & - & - & - & & \\
\hline & Control 2 & No treatment & - & - & - & & \\
\hline Koprivová et al., $2013^{\mathrm{a}}$ & $\begin{array}{l}\text { Treatment } \\
\text { Control }\end{array}$ & $\begin{array}{l}\text { EEG + CBT } \\
\text { EEG sham + CBT }\end{array}$ & Individualized EEG & 25.0 & 30 & Y-BOCS & $1.02[0.28 ; 1.75]$ \\
\hline Scheinost et al., $2014^{\mathrm{a}}$ & Treatment & fMRI & $\begin{array}{l}\text { BOLD from orbitofrontal/ } \\
\text { BA } 10\end{array}$ & 1.6 & - & Y-BOCS & $3.31[1.23 ; 5.40]$ \\
\hline \multirow[t]{2}{*}{ Deng et al., $2014^{\mathrm{a}}$} & Treatment & $\mathrm{EEG}+$ Drug + $\mathrm{CBT}$ & $\begin{array}{l}\text { EEG alpha, sensorimotor } \\
\text { rhythm, and theta }\end{array}$ & 40.0 & 24 & Y-BOCS & $6.23[4.80 ; 7.66]$ \\
\hline & Control & Drug + CBT & - & - & - & & \\
\hline Buyukturkoglu et al., $2015^{\mathrm{a}}$ & Treatment & fMRI & BOLD from anterior insula & 10.0 & - & Y-BOCS & $0.01[-0.64 ; 0.66]$ \\
\hline
\end{tabular}

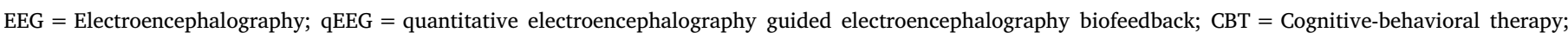

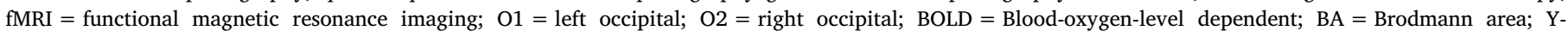
BOCS = Yale-Brown Obsessive Compulsive Scale; PI-WSUR = Padua Inventory-Washington State University Revision; CI = Confidence interval.

a studies included meta-analysis.

(Koprivová et al., 2013). The other works were performed in Turkey (Sürmeli and Ertem, 2011), Iran (Barzegary et al., 2011), Canada (Mills and Solyom, 1974), China (Deng et al., 2014), and USA (Hammond, 2004, 2003; LeVine, 1983; Scheinost et al., 2014). Three works were randomized controlled trials (Barzegary et al., 2011; Deng et al., 2014; Koprivová et al., 2013). From these 3 trials, 1 was a doubleblind study (Koprivová et al., 2013) and 1 was a single-blind study (Deng et al., 2014). The remaining studies were not controlled.

Table 1 shows a summary of the studies included in the systematic review. The total number of OCD patients enrolled in the biofeedback intervention was 102 and 53 OCD patients were included in control groups. The average age of the treated patients was 29.1 (6.9) years [mean (standard deviation)] and for the control patients was 28.7 (2.1) years. The biofeedback intervention group had a total of 61 females and 44 males [including a study with 3 dropout patients (Deng et al., 2014)] and the control group had 30 females and 27 males [including a study with 4 dropout patients (Deng et al., 2014)].

All studies applied an intervention based on neurofeedback with fMRI ( $n=2$ (Buyukturkoglu et al., 2015; Scheinost et al., 2014)) or EEG $(n=7)$, except one publication that used thermal biofeedback (LeVine, 1983). Two studies combined the neurofeedback effects with CBT (Koprivová et al., 2013) or medication and CBT (Deng et al., 2014). For control, the 3 trials mentioned above used a waiting list group and medication group (Barzegary et al., 2011), a medication and CBT combined group (Deng et al., 2014), or a sham biofeedback and CBT combined group (Koprivová et al., 2013). The biofeedback intervention had an average duration of 31.8 (21.8) sessions (one study with missing information (LeVine, 1983)). The brain regions target during the neurofeedback intervention were the anterior insula (Buyukturkoglu et al., 2015) and orbitofrontal cortex/Brodmann area 10 (Scheinost et al., 2014), but most of the studies used an individualized approach targeting subject-specific brain regions with abnormal activation (Barzegary et al., 2011; Hammond, 2003, 2004; Koprivová et al., 2013; Sürmeli and Ertem, 2011). The studies selected for the systematic review did not include overlap data from the same participant. Table 2 shows a summary of the intervention characteristics of the studies included in the systematic review.

The main outcome measure was the change in psychometric scales score associated with symptomatic manifestation. Results are presented for the Y-BOCS $(n=7)$ or Padua Inventory-Washington State
University Revision (PI-WSUR; $n=1$ (Rapp et al., 2016)) since both scales evaluate the severity of obsessive-compulsive symptoms (Table 2). Psychometric scales assessing other parameters (e.g. depression and anxiety) were used only once per study, thus, were excluded from the analysis. Two studies were excluded from the metaanalysis because they reported only qualitative outcomes (LeVine, 1983; Mills and Solyom, 1974). Additionally, 2 publications with small sample sizes ( $n=1$ (Hammond, 2004) and $n=2$ (Hammond, 2003)) were not included because the effect size could not be estimated. We also excluded another study due to the lack of information for statistical calculation (Barzegary et al., 2011) after contacting the authors without success. In conclusion, 10 studies were accepted for the systematic review and 5 for the meta-analysis.

\subsection{Risk of bias within studies}

Table 3 indicates the information regarding the quality of the studies included in the systematic review. Most of the works presented low quality for the evaluated parameters, except for sponsoring bias. Only the 3 controlled and randomized trials were considered moderate to high-quality publications (Barzegary et al., 2011; Deng et al., 2014; Koprivová et al., 2013). Nonetheless, 2 studies did not control for placebo effects (Barzegary et al., 2011; Deng et al., 2014). The third one was sponsored by a company and lacked a conflict of interests statement, thus, we considered only moderate quality regarding sponsoring bias (Koprivová et al., 2013).

\subsection{Results of individual studies}

All 5 studies considered for meta-analysis applied a neurofeedback intervention with OCD patients and used the Y-BOCS scale to measure symptomatic changes. Taking into account that only 2 (Deng et al., 2014; Koprivová et al., 2013) of the 5 studies selected were controlled and that distinct control groups were used, the effect size estimation was only performed within the intervention group - the standardized mean change of Y-BOCS scores from baseline to after neurofeedback (Table 2). Effect size calculation is represented in Table 2 and on the forest plot in Fig. 2. The within-group effect of neurofeedback treatment for OCD patients varied from small to large values for individual studies (McGrath and Meyer, 2006; Tomczak and Tomczak, 2014). 
Table 3

Summary of the quality assessment of the studies included in the systematic review.

\begin{tabular}{|c|c|c|c|c|c|}
\hline Study & Control & Randomization & Patients' blindness & Researchers' blindness & Sponsoring bias \\
\hline \multicolumn{6}{|l|}{ Mills et al., 1974} \\
\hline \multicolumn{6}{|c|}{ LeVine et al., 1983} \\
\hline \multicolumn{6}{|c|}{ Hammond et al., 2003} \\
\hline \multicolumn{6}{|c|}{ Hammond et al., 2004} \\
\hline \multicolumn{6}{|c|}{ Sürmeli et al., 2011} \\
\hline \multicolumn{6}{|c|}{ Barzegary et al., 2011} \\
\hline \multicolumn{6}{|c|}{ Kopřivová et al., 2013} \\
\hline \multicolumn{6}{|c|}{ Scheinost et al., 2014} \\
\hline \multicolumn{6}{|l|}{ Deng et al., 2014} \\
\hline \multicolumn{6}{|c|}{ Buyukturkoglu et al., 2015} \\
\hline Low quality; & & High o & e information. & & \\
\hline
\end{tabular}

\subsection{Synthesis of results}

The overall within-group effect size was 2.47 (CI 95\% 0.37; 4.57), a large effect size favoring the neurofeedback intervention for OCD patients (McGrath and Meyer, 2006; Tomczak and Tomczak, 2014). However, the CI pointed to a large standard error for this effect size. Moreover, the meta-analysis revealed a high heterogeneity value $[Q$ $(d f=4)=71.59, p<0.0001]$ corresponding to an elevated value of inconsistency $\left(I^{2}\right.$ ) of $96.64 \%$ (CI 95\% 90.01\%; 99.60\%) [Fig. 2].

\subsection{Risk of bias across studies}

The funnel plot in Fig. 3 indicates strong data asymmetry, possibly reflecting the existence of reporting bias, poor methodological designs, and the high heterogeneity values reported above (Sterne et al., 2011).

\subsection{Additional analyses}

Results from the exploratory meta-regression indicated that the heterogeneity values [ $Q$ residual $(d f=1)=37.35, p<0.0001$; $Q$ predictors $\quad(d f=3)=0.90, \quad p=0.82 ; \quad I^{2}=97.32 \% \quad$ (CI $\quad 95 \%$ $86.55 \%$; $>99.73 \%$ ] were not explained by the predictors. The average age $[B=0.09 ; Z=0.37 ; p=0.72]$, gender ratio $[B=-0.40$; $Z=-0.55 ; p=0.58]$, and mean session duration $[B=0.06 ; Z=0.63$; $p=0.53$ ] did not present a statistically significant effect on the model.

\section{Discussion}

Our work aimed to evaluate the evidence for therapeutic efficacy of biofeedback in OCD\&RD, albeit all publications selected pertain to OCD patients and mostly neurofeedback interventions. The results point to a positive effect of neurofeedback in OCD patients' symptomology but reveal serious limitations on the research procedures (e.g. lack of proper control groups and small sample sizes), high heterogeneity among studies, and a putative reporting bias. To conclude, further research with high-quality standards is needed to address the efficacy of neurofeedback in OCD. Additionally, biofeedback should be explored as a potential therapy for other OCD\&RD.

Body dysmorphic disorder, hoarding disorder, trichotillomania/ hair-pulling disorder, and excoriation/skin-picking disorder are related OCD diseases that may also benefit from biofeedback approaches (Abramowitz, 2018; Sachs and Erfurth, 2018), thus, more studies should be performed in the future. Additionally, other biofeedback interventions apart from neurofeedback (e.g. approaches using skin conductance, respiratory, or electrocardiogram signals) might have the potential to be used in ecological environments at lower costs (Schoenberg and David, 2014). Indeed, devices incorporating mobile technology are starting to be applied to help patients with psychiatric disorders in everyday life (Adams et al., 2017; Bhugra et al., 2017; Loo Gee et al., 2016; Van Ameringen et al., 2017; Versluis et al., 2016). Nonetheless, EEG neurofeedback is also a widely available technique that lacks the regional resolution of fMRI, especially for activity in deep/subcortical areas, but implicates lower costs and good temporal

Study

Size Weight (\%)

Effect size [Cl $95 \%]$

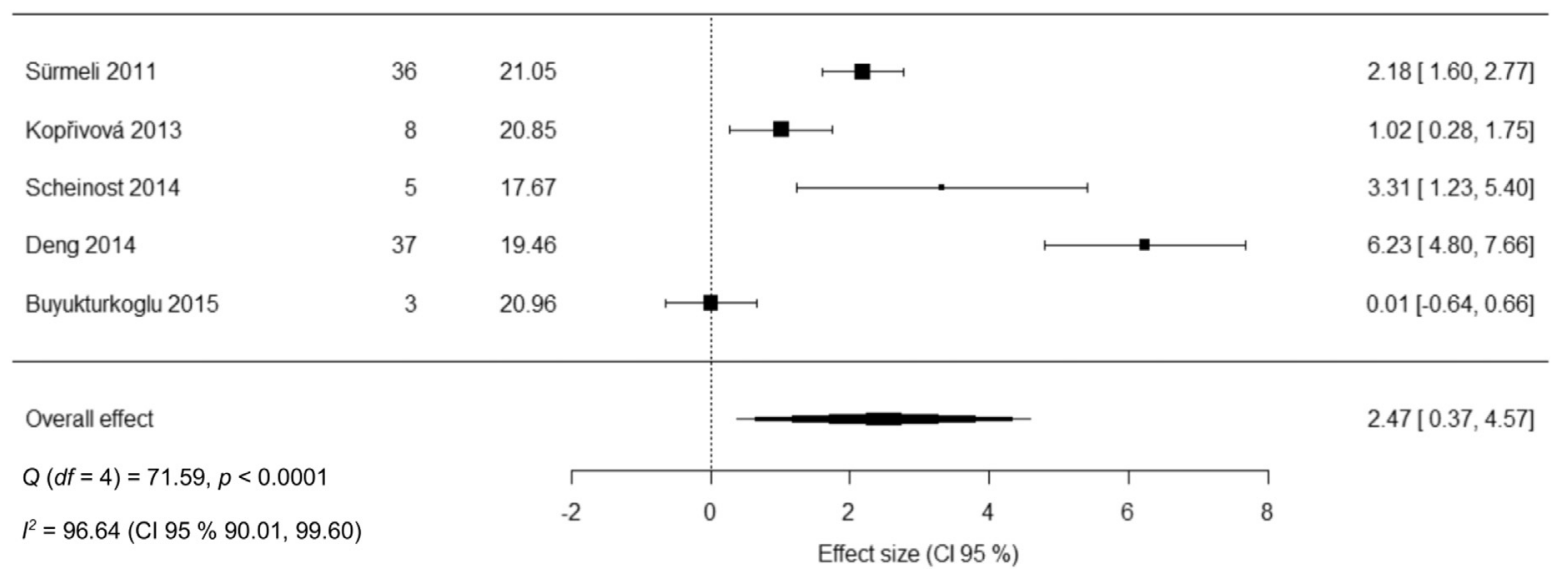

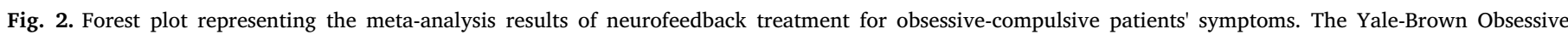

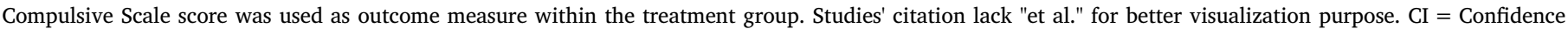
interval; $Q=$ Heterogeneity; $I^{2}=$ Inconsistency. 


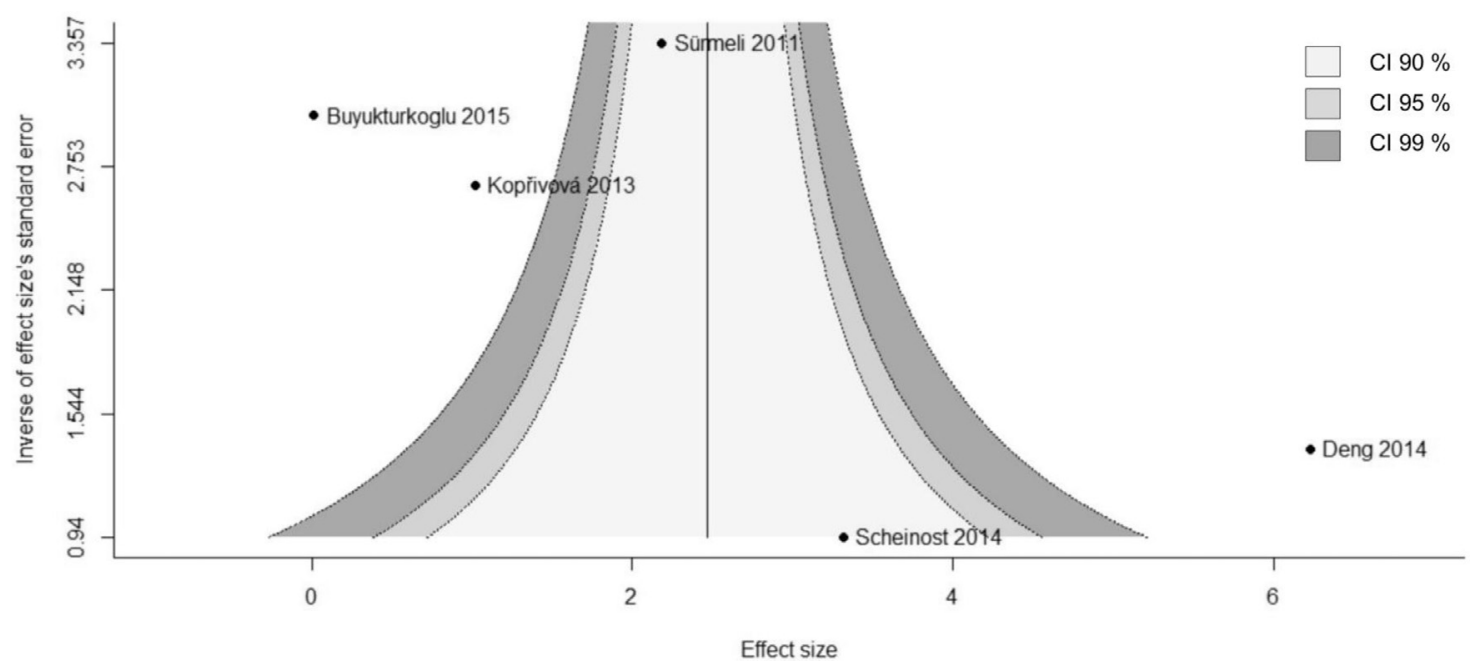

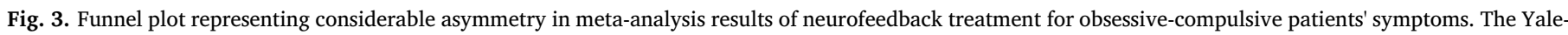

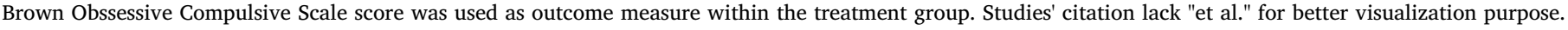
$\mathrm{CI}=$ Confidence interval.

resolution. fMRI has a better spatial resolution to localize brain regions but has low temporal resolution and entails high costs (Begemann et al., 2016; Sitaram et al., 2016; Thibault et al., 2018) .

Studies included merely for the systematic review presented a reduction in OCD symptoms after neurofeedback (Barzegary et al., 2011; Hammond, 2004, 2003; LeVine, 1983; Mills and Solyom, 1974). Regarding the meta-analysis results, the overall effect of neurofeedback in the symptomatology of OCD patients was high. However, this value concerns only changes in the intervention group (within-group analysis). Only two studies from the meta-analysis were controlled (Deng et al., 2014; Koprivová et al., 2013) and the type of control group was distinct, preventing a conclusive between-group analysis on the effect size. Nonetheless, these two trials were included in a previous review (Begemann et al., 2016) regarding EEG neurofeedback effects in psychiatric diseases, where authors reported a moderate to high betweengroup effect mainly for compulsive symptoms. However, Kopřivová et al. (2013) was the only study accounting for placebo effects by using a sham neurofeedback control group. Since patients are always aware of the neurofeedback intervention, it is crucial to have placebo (Arns et al., 2017; Rogala et al., 2016; Sitaram et al., 2016) or/and active controlled studies. Indeed, some reports point that active comparators such as medication or CBT might be better than sham groups because psychiatric treatments usually involve a placebo component that might positively affect the outcomes (Geddes and Cipriani, 2015; Hammond, 2011; Pigott et al., 2018).

Another drawback of the selected studies was the small sample size. For the meta-analysis, the sample size ranged from 3 to 37 patients with more than half of the studies including less than 10 patients. Additionally, more women than men were included ( 54 women versus 38 men). Previous research indicated that OCD male patients show a higher vulnerability for more severe disease manifestation (Goldberg et al., 2015). Moreover, OCD is a very heterogeneous disease with different symptomatic dimensions (contamination/cleaning, symmetry/repeating/ordering/counting, sexual/religious/aggressive, and harming) (American Psychiatric Association, 2013). Thus, patients might respond differently to biofeedback interventions depending on obsession and compulsion categories (Thorsen et al., 2018) and gender. Some of the studies included in our review lack information about symptomatic dimensions of patients and the diagnosis instruments. Future studies should provide clear information about patients' diagnosis and symptoms to better characterize biofeedback outcomes. Furthermore, more than half of the studies included OCD patients with comorbidities which may have an influence on intervention outcomes.
All these factors might have contributed to the high level of heterogeneity among studies. Lastly, the selected studies lack clear information regarding the proportion of responder patients based on specific criteria (except (Deng et al., 2014)) and secondary effects of neurofeedback. Moreover, the heterogeneity may also arise from the inclusion of studies with different neurofeedback modalities (fMRI and EEG) and distinct target brain regions. More studies were performed with fMRI than EEG feedback possibly because fMRI is a less available, less practical, newer, and more expensive technique. Indeed, the two studies using fMRI neurofeedback have a low sample size possibly indicating that these were pilot works.

The reported effect size was not associated with the number of neurofeedback sessions and the sample characteristics (age and gender ratio). This conclusion must be interpreted with caution given the small number of studies included in the meta-regression and that the number of sessions may be a biased measure of the intervention duration (these results may be false negatives). Unfortunately, not all the studies reported session duration values, preventing a real estimation of the intervention length. Future publications should clearly describe the intervention protocol to allow replication.

In clinical terms, neurofeedback might have the potential for treatment-resistant patients to minimize prolonged treatment periods and the use of invasive therapies such as deep brain stimulation and radiosurgery (Micoulaud-Franchi et al., 2015; Seibell and Hollander, 2014). Additionally, EEG portable systems and mobile devices are being developed to allow a more ecological biofeedback approach in the future (Enriquez-Geppert et al., 2017). Indeed, recent findings with OCD and Tourette Syndrome patients suggest that fMRI neurofeedback leads to a continuous symptomatic improvement even weeks after the end of the intervention. Thus, more ecological techniques might imply longer biofeedback effects. These authors claim that patients might adopt the skills learned during neurofeedback in a similar way to CBT (Rance et al., 2018). A recent meta-analysis addressing the therapeutic effect of CBT in OCD symptoms revealed a medium to large effect size (Carpenter et al., 2018). However, this work was conducted with four randomized placebo-controlled trials, thus, results cannot be compared to ours. Another meta-analysis (Sugarman et al., 2017) of thirteen trials evaluating the therapeutic outcomes of several antidepressant drugs for OCD also reported a medium to a large effect size of the medication without considering the placebo control. Although our review shows a similar effect size, the included studies have patients already exposed to medication and, some of them, psychotherapy. Despite the implementation of washout periods before neurofeedback in some 
publications, the use of other treatment approaches might have influenced the results. Future works should consider previous/current treatments as a potential confounding variable.

\subsection{Limitations}

The search methodology was performed by a sole author (SF), although a strict procedure was followed accordingly to PRISMA guidelines (Liberati et al., 2009; Moher et al., 2009).

We included non-randomized and non-controlled studies to provide a complete and clinically helpful overview of biofeedback interventions for OCD\&RD. This process enabled us to highlight flaws and novel research approaches for future works.

Our results may have been influenced by publication bias. Our search was limited by articles published in English and one publication was not available for full-text reading. No unpublished studies were found, but our search was limited to four databases. Nonetheless, the analysis of reference lists allowed a more exhaustive search. Additionally, three publications were not selected for meta-analysis due to incomplete or qualitative reporting of outcomes possibly increasing reporting bias (Barzegary et al., 2011; LeVine, 1983; Mills and Solyom, 1974). Lastly, the new categorization of OCD\&RD in DSM-5 might prevent the retrieval of articles following the last classification. However, our search was conducted with specific disorder names to avoid missing information.

\subsection{Conclusions}

In summary, the poor methodological quality of the select studies prevents evidence-based conclusions on the efficacy of neurofeedback in the treatment of OCD. Moreover, the lack of studies addressing other biofeedback interventions and OCD related disorders highlights the need for further research. Thus, CBT and drug therapy remain at the forefront of OCD\&RD treatment.

Future studies should follow high-quality guidelines namely predetermined sample sizes (with power calculation), randomization and blinding, selection of proper control groups, a complete clinical characterization of patients, and a clear description of biofeedback protocols and study outcomes.

\section{Declarations of interest}

None.

\section{Acknowledgments}

We would like to thank Pedro Moreira for the help with the statistical analysis.

\section{Funding}

This work has been funded by FEDER funds, through the Competitiveness Factors Operational Programme (COMPETE), and by National funds, through the Foundation for Science and Technology (FCT), under the scope of the project POCI-01-0145-FEDER-007038. This article has been developed under the scope of the project NORTE01-0145-FEDER-000013, supported by the Northern Portugal Regional Operational Programme(NORTE 2020), under the Portugal 2020 Partnership Agreement, through the European Regional Development Fund (FEDER), and the BIAL foundation, Porto, Portugal (grant number PT/FB/BL-2016-206). SF was supported by a combined Ph. D. scholarship from FCT and the company iCognitus4ALL - IT Solutions, Lda, Braga, Portugal (grant number PD/BDE/127839/2016).

\section{Supplementary materials}

Supplementary material associated with this article can be found, in the online version, at doi:10.1016/j.psychres.2018.12.096.

\section{References}

Abramowitz, J.S., 2018. Presidential address: are the obsessive-compulsive related disorders related to obsessive-compulsive disorder? A critical look at DSM-5's new category. Behav. Ther 49, 1-11. https://doi.org/10.1016/j.beth.2017.06.002.

Adams, Z.W., McClure, E.A., Gray, K.M., Danielson, C.K., Treiber, F.A., Ruggiero, K.J. 2017. Mobile devices for the remote acquisition of physiological and behavioral biomarkers in psychiatric clinical research. J. Psychiatr. Res. 85, 1-14. https://doi. org/10.1016/j.jpsychires.2016.10.019.

American Psychiatric Association, 2013. Diagnostic and statistical manual of mental disorders, (5th ed.). American Psychiatric Publishing, Arlington, VA.

Arns, M., Batail, J.-M., Bioulac, S., Congedo, M., Daudet, C., Drapier, D., et al., 2017. Neurofeedback: one of today's techniques in psychiatry? Encephale. 43, 135-145. https://doi.org/10.1016/j.encep.2016.11.003.

Barzegary, L., Yaghubi, H., Rostami, R., 2011. The effect of QEEG- guided neurofeedback treatment in decreasing of OCD symptoms. Procedia - Soc. Behav. Sci. 30 , 2659-2662. https://doi.org/10.1016/j.sbspro.2011.10.519.

Begemann, M.J.H., Florisse, E.J.R., van Lutterveld, R., Kooyman, M., Sommer, I.E., 2016 Efficacy of EEG neurofeedback in psychiatry: a comprehensive overview and metaanalysis. Transl. Brain Rhythm. 1, 19-29. https://doi.org/10.15761/TBR.1000105.

Bhugra, D., Tasman, A., Pathare, S., Priebe, S., Smith, S., Torous, J., et al., 2017. The WPA- lancet psychiatry commission on the future of psychiatry. Lancet Psychiatry 4 , 775-818. https://doi.org/10.1016/S2215-0366(17)30333-4.

Bruin, W., Denys, D., van Wingen, G., 2018. Diagnostic neuroimaging markers of obsessive-compulsive disorder: Initial evidence from structural and functional MRI studies. Prog. Neuro-Psychopharmacology Biol. Psychiatry In Press. https://doi.org/ 10.1016/j.pnpbp.2018.08.005.

Buyukturkoglu, K., Roettgers, H., Sommer, J., Rana, M., Dietzsch, L., Arikan, E.B., et al., 2015. Self-regulation of anterior insula with real-time fMRI and its behavioral effects in obsessive-compulsive disorder: a feasibility study. PLoS One 10, e0135872. https://doi.org/10.1371/journal.pone.0135872.

Carpenter, J.K., Andrews, L.A., Witcraft, S.M., Powers, M.B., Smits, J.A.J., Hofmann, S.G., 2018. Cognitive behavioral therapy for anxiety and related disorders: a meta-analysis of randomized placebo-controlled trials. Depress. Anxiety 1-13. https://doi.org/10. 1002/da.22728.

Deng, X., Wang, G., Zhou, L., Zhang, X., Yang, M., Han, G., et al., 2014. Randomized controlled trial of adjunctive EEG-biofeedback treatment of obsessive-compulsive disorder. Shanghai Arch. psychiatry 26, 272-279. https://doi.org/10.11919/j.issn. 1002-0829.214067.

Enriquez-Geppert, S., Huster, R.J., Herrmann, C.S., 2017. EEG-Neurofeedback as a tool to modulate cognition and behavior: a review tutorial. Front. Hum. Neurosci. 11, 1-19. https://doi.org/10.3389/fnhum.2017.00051.

Geddes, J.R., Cipriani, A., 2015. Time to abandon placebo control in pivotal phase III trials? World Psychiatry 14, 306-307. https://doi.org/10.1002/wps.20246.

Goldberg, X., Soriano-Mas, C., Alonso, P., Segalàs, C., Real, E., López-Solà, C., et al., 2015. Predictive value of familiality, stressful life events and gender on the course of obsessive-compulsive disorder. J. Affect. Disord. 185, 129-134. https://doi.org/10. 1016/j.jad.2015.06.047.

Gonçalves, Ó., Camargo Batistuzzo, M., Sato, J., 2017. Real-time functional magnetic resonance imaging in obsessive-compulsive disorder. Neuropsychiatr. Dis. Treat. 13, 1825-1834. https://doi.org/10.2147/NDT.S121139.

Hammond, D.C., 2011. Placebos and neurofeedback: a case for facilitating and maximizing Placebo response in neurofeedback treatments. J. Neurother. 15, 94-114. https://doi.org/10.1080/10874208.2011.570694.

Hammond, D.C., 2004. Treatment of the obsessional subtype of obsessive compulsive disorder with neurofeedback. Biofeedback 32, 9-12.

Hammond, D.C., 2003. QEEG-guided neurofeedback in the treatment of obsessive compulsive disorder. J. Neurother. 7, 25-52. https://doi.org/10.1300/J184v07n02_03.

Hardy, R.J., Thompson, S.G., 1998. Detecting and describing heterogeneity in metaanalysis. Stat. Med. 17, 841-856 https://doi.org/10.1002/(SICI)10970258(19980430)17:8<841::AID - SIM781 > 3.0.CO;2-D.

Higgins, J.P.T., Green, S. (Eds.), 2011. Cochrane Handbook for Systematic Reviews of Interventions Version 5.1.0 [updated March 2011]. The Cochrane Collaboration. Available from http://handbook.cochrane.org.

Higgins, J.P.T., 2003. Measuring inconsistency in meta-analyses. BMJ 327, 557-560. https://doi.org/10.1136/bmj.327.7414.557.

Higgins, J.P.T., Thompson, S.G., 2002. Quantifying heterogeneity in a meta-analysis. Stat. Med. 21, 1539-1558, https://doi.org/10.1002/sim.1186.

Hozo, S.P., Djulbegovic, B., Hozo, I., 2005. Estimating the mean and variance from the median, range, and the size of a sample. BMC Med. Res. Methodol. 5, 13. https://doi. org/10.1186/1471-2288-5-13.

Koprivová, J., Congedo, M., Raszka, M., Prasko, J., Brunovský, M., Horácek, J., 2013. Prediction of treatment response and the effect of independent component neurofeedback in obsessive-compulsive disorder: a randomized, sham-controlled, doubleblind study. Neuropsychobiology 67, 210-223. https://doi.org/10.1159/000347087.

LeVine, W.R., 1983. Behavioral and biofeedback therapy for a functionally impaired musician: a case report. Biofeedback Self. Regul. 8, 101-107.

Liberati, A., Altman, D.G., Tetzlaff, J., Mulrow, C., Gøtzsche, P.C., Ioannidis, J.P.A., et al., 2009. The PRISMA statement for reporting systematic reviews and meta-analyses of 
studies that evaluate health care interventions: explanation and elaboration. PLoS Med 6, e1000100. https://doi.org/10.1371/journal.pmed.1000100.

Loo Gee, B., Griffiths, K.M., Gulliver, A., 2016. Effectiveness of mobile technologies delivering ecological momentary interventions for stress and anxiety: a systematic review. J. Am. Med. Informatics Assoc. 23, 221-229. https://doi.org/10.1093/jamia/ ocv043.

McGrath, R.E., Meyer, G.J., 2006. When effect sizes disagree: the case of $\mathrm{r}$ and d. Psychol. Methods 11, 386-401. https://doi.org/10.1037/1082-989X.11.4.386.

Micoulaud-Franchi, J.-A., McGonigal, A., Lopez, R., Daudet, C., Kotwas, I., Bartolomei, F., 2015. Electroencephalographic neurofeedback: level of evidence in mental and brain disorders and suggestions for good clinical practice. Neurophysiol. Clin. Neurophysiol. 45, 423-433. https://doi.org/10.1016/j.neucli.2015.10.077.

Mills, G.K., Solyom, L., 1974. Biofeedback of EEG alpha in the treatment of obsessive ruminations: an exploration. J. Behav. Ther. Exp. Psychiatry 5, 37-41. https://doi. org/10.1016/0005-7916(74)90011-1.

Moher, D., Liberati, A., Tetzlaff, J., Altman, D.G., 2009. Preferred reporting items for systematic reviews and meta-analyses: the PRISMA statement. PLoS Med 6, e1000097. https://doi.org/10.1371/journal.pmed.1000097.

Moreira, P.S., Marques, P., Soriano-Mas, C., Magalhães, R., Sousa, N., Soares, J.M., et al., 2017. . The neural correlates of obsessive-compulsive disorder: a multimodal perspective. Transl. Psychiatry 7, e1224. https://doi.org/10.1038/tp.2017.189.

Morris, S.B., 2008. Estimating effect sizes from pretest-posttest-control group designs. Organ. Res. Methods 11, 364-386. https://doi.org/10.1177/1094428106291059.

Morris, S.B., DeShon, R.P., 2002. Combining effect size estimates in meta-analysis with repeated measures and independent-groups designs. Psychol. Methods 7, 105-125. https://doi.org/10.1037//1082-989X.7.1.105.

Nakagawa, S., Cuthill, I.C., 2007. Effect size, confidence interval and statistical significance: a practical guide for biologists. Biol. Rev. 82, 591-605. https://doi.org/10. 1111/j.1469-185X.2007.00027.x.

Pearcy, C.P., Anderson, R.A., Egan, S.J., Rees, C.S., 2016. A systematic review and metaanalysis of self-help therapeutic interventions for obsessive-compulsive disorder: Is therapeutic contact key to overall improvement? J. Behav. Ther. Exp. Psychiatry 51, 74-83. https://doi.org/10.1016/j.jbtep.2015.12.007.

Pigott, H.E., Cannon, R., Trullinger, M., 2018. The fallacy of Sham-controlled neurofeedback trials: a reply to Thibault and Colleagues (2018). J. Atten. Disord 108705471879080. https://doi.org/10.1177/1087054718790802.

Rance, M., Walsh, C., Sukhodolsky, D.G., Pittman, B., Qiu, M., Kichuk, S.A., et al., 2018. Time course of clinical change following neurofeedback. Neuroimage 181, 807-813. https://doi.org/10.1016/j.neuroimage.2018.05.001.

Rapp, A.M., Bergman, R.L., Piacentini, J., Mcguire, J.F., 2016. Evidence-based assessment of obsessive-compulsive disorder. J. Cent. Nerv. Syst. Dis. 8 JCNSD.S38359. https:// doi.org/10.4137/JCNSD.S38359.

Rogala, J., Jurewicz, K., Paluch, K., Kublik, E., Cetnarski, R., Wróbel, A., 2016. The do's and don'ts of neurofeedback training: a review of the controlled studies using healthy adults. Front. Hum. Neurosci. 10, 1-12. https://doi.org/10.3389/fnhum.2016. 00301.

Sachs, G., Erfurth, A., 2018. Obsessive compulsive and related disorders: from the biological basis to a rational pharmacological treatment. Int. J. Neuropsychopharmacol. 21, 59-62. https://doi.org/10.1093/ijnp/pyx101.

Scheinost, D., Stoica, T., Wasylink, S., Gruner, P., Saksa, J., Pittenger, C., et al., 2014. Resting state functional connectivity predicts neurofeedback response. Front. Behav. Neurosci 8, 338. https://doi.org/10.3389/fnbeh.2014.00338.

Schoenberg, P.L.A., David, A.S., 2014. Biofeedback for psychiatric disorders: a systematic review. Appl. Psychophysiol. Biofeedback 39, 109-135. https://doi.org/10.1007/ s10484-014-9246-9.

Seibell, P., Hollander, E., 2014. Management of obsessive-compulsive disorder. F1000Prime Rep. 6, 1-6. https://doi.org/10.12703/P6-68.

Simon, D., Kaufmann, C., Kniesche, R., Kischkel, E., Kathmann, N., 2013. Autonomic responses and neural-cardiac coupling during individually tailored symptom provocation in obsessive-compulsive disorder. J. Anxiety Disord. 27, 635-644. https://doi. org/10.1016/j.janxdis.2013.08.005.

Sitaram, R., Ros, T., Stoeckel, L., Haller, S., Scharnowski, F., Lewis-Peacock, J., et al., 2016. Closed-loop brain training: the science of neurofeedback. Nat. Rev. Neurosci. 18, 86-100. https://doi.org/10.1038/nrn.2016.164.

Sterne, J.A.C., Sutton, A.J., Ioannidis, J.P.A., Terrin, N., Jones, D.R., Lau, J., et al., 2011. Recommendations for examining and interpreting funnel plot asymmetry in metaanalyses of randomised controlled trials. BMJ 343 d4002-d4002. https://doi.org/10 1136/bmj.d4002.

Sugarman, M.A., Kirsch, I., Huppert, J.D., 2017. Obsessive-compulsive disorder has a reduced placebo (and antidepressant) response compared to other anxiety disorders: a meta-analysis. J. Affect. Disord. 218, 217-226. https://doi.org/10.1016/j.jad.2017. 04.068.

Sürmeli, T., Ertem, A., 2011. Obsessive compulsive disorder and the efficacy of qEEGguided neurofeedback treatment: a case series. Clin. EEG Neurosci. 42, 195-201. https://doi.org/10.1177/155005941104200310.

Thibault, R.T., MacPherson, A., Lifshitz, M., Roth, R.R., Raz, A., 2018. Neurofeedback with fMRI: a critical systematic review. Neuroimage 172, 786-807. https://doi.org/ 10.1016/j.neuroimage.2017.12.071.

Thompson, S.G., Higgins, J.P.T., 2002. How should meta-regression analyses be undertaken and interpreted? Stat. Med. 21, 1559-1573. https://doi.org/10.1002/sim. 1187.

Thorsen, A.L., Kvale, G., Hansen, B., van den Heuvel, O.A., 2018. Symptom dimensions in obsessive-compulsive disorder as predictors of neurobiology and treatment response. Curr. Treat. Options Psychiatry. https://doi.org/10.1007/s40501-018-0142-4.

Tomczak, M., Tomczak, E., 2014. The need to report effect size estimates revisited. An overview of some recommended measures of effect size. Trends Sport Sci. 1, 19-25.

Van Ameringen, M., Turna, J., Khalesi, Z., Pullia, K., Patterson, B., 2017. There is an app for that! The current state of mobile applications (apps) for DSM-5 obsessive-compulsive disorder, posttraumatic stress disorder, anxiety and mood disorders. Depress. Anxiety 34, 526-539. https://doi.org/10.1002/da.22657.

Versluis, A., Verkuil, B., Spinhoven, P., van der Ploeg, M.M., Brosschot, J.F., 2016. Changing mental health and positive psychological well-being using ecological momentary interventions: a systematic review and meta-analysis. J. Med. Internet Res. 18, e152. https://doi.org/10.2196/jmir.5642. 